\title{
Computed Tomography Image-guided Interaoperative and Stereotactic Calculation Method
}

\author{
Tareq Alhadidi \\ Medical Equipment Technology Department, College of Applied Medical Sciences, Prince Sattam bin \\ Abdulaziz University, Alkharj, Saudi Arabia \\ hadidi2@hotmail.com
}

\begin{abstract}
Targeting of deep brain structure pathologies in the operation room and the concept of stereotactic that is applied on the affected zone is described. The achievement of a high accuracy using specialized stereotactic apparatus which controls the surgical tool movement inside the brain is discussed. Calculation of algorithms is necessary for operational planning included. Stereotactic system through combination of hardware-software package which specify the coordinates of surgical zone is designed. Using stereotactic method landmarks during execution of intra-operation by using CT and digital image segmentation are approved and analyzed.
\end{abstract}

Keywords: Computerized tomography; Physical modeling; Stereotactic method; Stereotactic landmarks; Neurosurgery Simulation.

\section{Introduction}

Parkinson's disease is a chronic mobility disorder disease. In 1912, the Lirche carried out a kind of cervical rhizotomy in order to treat Parkinsonian tremor. Consequently, several attempts of surgical intervention to control certain types of movement disorders [1-3]. Other specialists also investigated several methods that target the basal ganglia to treat locomotion disorders $[4,5]$.

The stereotactic surgery was well-established conceptually in advance to the wide utility of several stereotactic frame apparatuses $[7,8]$. Furthermore, the Russian anatomist Zernov [6] innovated a map of the human intra-cerebral cortex in 1890 interrelated with the main brain functional fields. The basic function of stereotactic apparatus depends on the concept of local effect on a certain intra-cerebral target. This concept can be considered as using specialized stereotactic equipment, intra-scope visualization system, and calculation algorithms can be used for preoperative planning purposes. For getting the perfect performance of stereotactic equipment, first of all we have to perform accurate geometrical parameters calculation "target's coordinates" for the surgical intervention zone.

Before the advent of reconstructive techniques of brain mapping, the initial data for performing stereotactic calculations were components of cerebral ventricles system: this initial data obtained during the carrying out of orthogonal ventriculography in the frontal and sagittal projections [9, 10, and 13]. The geometrical constructions and the calculations include: (1) the calculation of stereotactic 
coordinates of intra-cerebral system using brain ventricle landmarks system, (2) modeling of surgical intervention zone by anatomical slices from specialized stereotactic atlases [18], (3) setting of control parameters of the stereotactic apparatus, and (4) calculation of the correction coefficient to reduce the distortion of X-ray images.

Although the universal use of a mentioned technique, this method is somewhat invasive, therefore, from the mid-eighties of the last century, attempts were made to use computerized tomography (CT) and magnetic resonance imaging (MRI) for stereotactic targeting (coordinate determination) [11-17], but the task is complicated by the fact that on the CT-images, it is impossible to conduct differential visualization of sub-cortical structures. The mechanism of scanning and image formation complicates determining standard stereotactic landmarks (and that is necessary for carrying out the calculations). Using of a "new" stereotactic landmarks method which contrastingly visible on topographic slices, often cause accuracy reduction of surgical intervention zone calculation [15].

Therefore, the main issue at the present stage is to solve the problem of adaptation of high informative equipment for intra-cerebral structures mapping, and make the methods of stereotactic calculations based on the brain ventricle landmarks generally accepted.

In this study, there is a description about a universal technique of stereotactic calculations using computed tomography as a tool for intra-operation visualization based on the stereotactic surgical systems applied to 27 patients with "Parkinson's disease", the stereotactic surgical system allows for operative maneuvers to be simulated on a simulation software before being implemented on the actual surgical intervention in the operation room. The three-dimensional surgical navigation improves surgical accuracy and can help convert a virtual surgical plan to the operative setting.

\section{Experiments and Analysis}

CT stereotactic calculations are performed on the basis of neurosurgical department of the Kharkov Regional Hospital while executing surgical intra-operation used a computed tomography CT MAX 3000 General Electric and stereotactic apparatus designed by Edward I. Kandel which allow modeling of polar coordinate system (two angles and one free translational degree for surgical instrument movement). When scanning, the patient lying in supine position, arms resting along the body, the head must secure well in the head holder, support lower legs, and the CT scan protocols is: Sequence Scan mode, Rotation time is $1.0 \mathrm{~s}$, Scan time is $1.0 \mathrm{~s}$. The stereotactic apparatus fastening is carried out through a fixation of carrying platform inside $25 \mathrm{~mm}$ diameter burr hole by using a collar clamp (in our work we did not use massive metal mounting frame).

\subsection{The work task}

The stereotactic calculation is considered as a high-precision guidance of a surgical instrument to the stereotactic target. For this purpose, the following must be considered:

* Create a virtual model of the human brain for neurosurgical simulation.

* Matching of brain coordinate systems, CT-slice and stereotactic apparatus.

* Determination of reference landmarks on intra-scope images.

* Formation of intra-cerebral stereotactic coordinate system.

* Calculation of the geometric characteristics of the surgical intervention zone. 
Tareq Alhadidi; Computed Tomography Image-guided Interaoperative and Stereotactic Calculation Method. Journal of Biomedical Engineering and Medical Imaging, Volume 5, No 1, February (2018), pp 1-10

* Formation of control parameters for the stereotactic apparatus.

Also while matching the coordinate systems at the initial stage of the procedure; it is necessary to strictly fix the patient's head in the apparatus's scanner gantry by using the following conditions:

i. Stereotactic apparatus platform is mounted perpendicularly to the sagittal or frontal plane of CT-images coordinate system.

ii. Fixation of X-ray contrast marks on the patient's head to ensure of the parallel scanning position of orbit meatal plane.

iii. Determination of craniography obtained by frontal and sagittal projections.

CT-slices under the above scanning conditions are shown in Fig. 1. (Divergence Compensation of X-rays is carried out with software developed by our team). CT-slices on sagittal (Cg) and frontal (F) planes are shown, respectively, in (a) and (b).

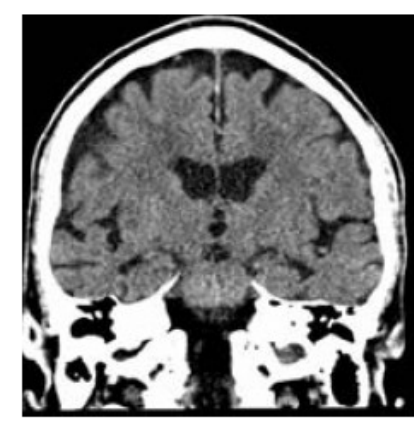

A)

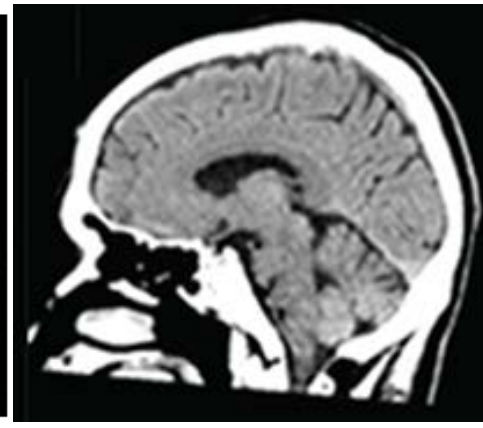

B)

Figure 1. CT-slices calculation.

\subsubsection{Subsystem visualization and target recognition}

Parkinson's disease structure which will be a subject of destruction or simulation (target) is characterized by metastasis state (cervical lymph node). The contrast of this structure is unclear and cannot be visualized from CT-slice using standard method. In this case, using indirect method of visualization for surgical zone (Parkinson's disease), will link the coordinate zone of the target to a reference landmark points inside the center of the brain (the central point between a line that connect anterior-posterior points of the III ventricle). For that we developed an algorithm for finding the central intra-cerebral coordinate system as our reference points which are used for targeting zone by average values. Usually for such landmarks anterior (CA) and posterior (CP) intra-cerebral white adhesions are used (Fig. 3(b)) which are typically defined by III ventricle contours in sagittal and frontal projections.

The basis by scanning is the orbit meatal line (o-m) (skull plane figure 3,a ) which passes through the line connects the posterior edge and external auditory meatus parallel to the middle line of (CA-CP) adhesion (by angle of deviation less than $5^{\circ}$ ). Therefore, from the CT configuration of the III ventricle (V3) (see Fig. 2a), the calculation method for (CA and CP) using axial slice is seen as a sequential scanning of it is region with $1 \mathrm{~mm}$ step, paralleled to orbit meatal plane. The target (V3) is the length measuring and identification of the slice which contains the second local zone (when identified from top) of the minimum local zone length of (V3) [slide number 3 in Fig. 2 (b)]. In accordance to the scan conditions, 
the horizontal CT-slice which contains a specified local minimum length of (V3) will be located in the stereotactic horizontal zero plane.

\subsubsection{Determination of (CA and CP) line:}

Thus, the task of contrasting landmark determination (CA and CP) will lead us to (V3) length calculation (the distance between anterior and posterior points of V3 contour center) in the studied and selected slice, which contains the second local zone (when identified from the top) of the minimum local zone length of (V3). For stereotactic coordinates CA (FCA, CgCA, GCA) and CP (FCP, CgCP, GCP) we take the coordinates of a central point sequentially to the top and bottom of the current slice contours (V3), taking in account the importance of finding the correct location of (CA and CP). In order of objective evaluation for (V3) length determination we use automatic algorithm for contour recognition based on image processing (segmentation and computer morphometry), for differentiating contours by thresholding image segmentation of target region (Fig. 3(a)) and construction of a binary function characteristic (Fig. 3(b)) by applying differential calculations for V3, and finally we get a colorless zone with clearly contour, and these latter considered as our reference point (Fig. 3(c)).

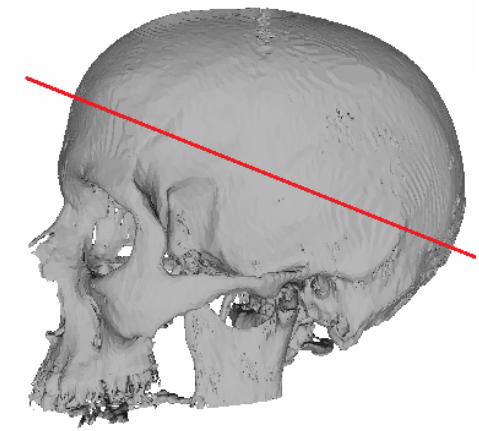

Fig. 2a.

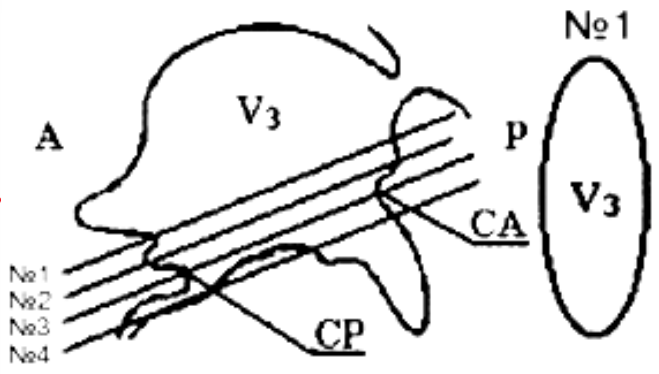

Fig. 2b.

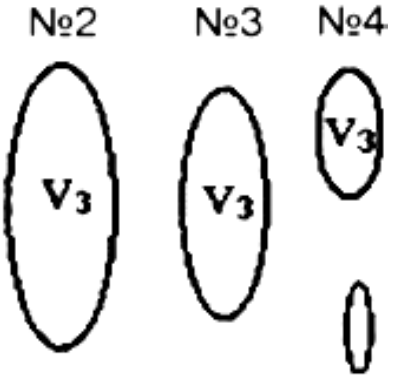

Fig. 2c.

Fig. 2a- Scanning technique used for orbit mental line calculation, $2 \mathrm{~b}$ - Configuration topological anatomical graph for (V3) finding in sagittal plane, 2c- Horizontal slide parallel to orbit meatal plane.

III ventricle contour determination:

The captured image contains many false contour objects and is extremely difficult for logical interpretation. In this work a combined method is applied to edge the contour: 1) by separating the object from the background, 2) by using of a logical filtration and 2-dimensional differentiation for the resulting 2-D image. Based on the fact that the area of interest (see Fig. 2b) contains one large object (with low contrast) on the color background, the segmentation is carried out by the method of threshold binarization. The histogram of such image (see Fig. 2c) has clearly pronounced bimodal character and has interactively threshold (can be execution manually) is not difficult. The Automation of such histogram analysis in order to select the two largest maxima peaks is not a trivial task and was carried out using the following algorithm:

1. smoothing the Linearity of the histogram for the purpose of blocking minor vibrations by using the following formula: 


$$
\overline{S_{i}}=\frac{1}{K+1} \sum_{j=-K}^{K} S_{i+j}, 1+K \leq i \leq 255-K,
$$

Where $S_{i}$ - histogram values at $i^{\text {th }}$ contrast level, $\bar{S}_{i}$ - averaged values, $K$ - number of previous and subsequent points near the point with an index $i$. The smoothing effectiveness when studying the contrast data distributions was implemented at the values of $K \geq 7$.

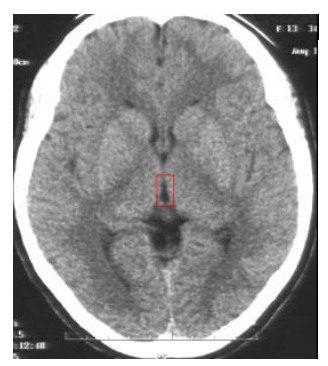

Fig.3a.

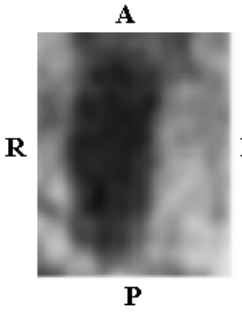

Fig. 3b.

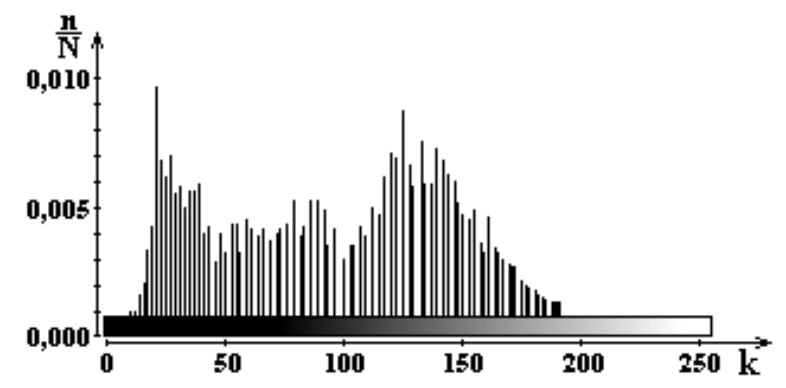

Fig. 3c.

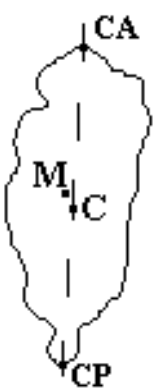

Fig. 3d.

Figure. 3 figure shows an automatic algorithm for (V3) finding and contour recognition.

(a) Axial tomographic slice at zero stereotactic horizontal plane,

(b) Target zone;

(c) Histogram of the processed area;

(d) Resulting contour image of (V3).

2. Finding local maxima in the smoothed histogram according to the following condition:

$$
\max _{i \in[0 ; 255]}\left\{\begin{array}{l}
\bar{s}_{0} \geq \bar{s}_{i+1} ; \\
\vec{s}_{i-1} \leq \bar{s}_{i} \geq \bar{s}_{i+1}, \quad 1 \leq i \leq 254 ; \\
\bar{s}_{255} \geq \bar{s}_{i-1} .
\end{array}\right\}
$$

By introducing additional conditions to the algorithm we can find a maximums flat peak on the smoothed histogram.

The threshold is selected as the mean value between the two maxima (the standard one and the maximum local, it is not located near the standard maximum). The false objects are eliminated with the help of a logical filter, taking into account the connectivity and regions size.

The resulting 2-D image that contains the object and the background was obtained using 2-dimensional differentiation operators based on the tabulating the calculation of the gradient $\mathrm{G}$, specified by contrast function $f(x, y)$ :

$$
\|G\|=|f(x+1, y)-f(x, y)|+|f(x, y+1)-f(x, y)|
$$


(CA-CP) centering:

After that, constructing the straight line connecting the two spikes $\mathrm{CA}-\mathrm{CP}$, and finding the coordinates center of (V3) mass $\mathrm{M}\left({ }^{X_{M}}, y_{M}\right)$ is done. This coordinates are determined by the formula:

$$
x_{M}=\frac{\sum_{i=1}^{N_{O}} x_{i}}{N_{o}} \quad y_{M}=\frac{\sum_{i=1}^{N_{O}} y_{i}}{N_{o}}
$$

Where $N_{o}$ - the number of points belongs to the object (V3).

The center of intra-cerebral stereotactic coordinate system is point $\mathrm{C}(\mathrm{Fc}, \mathrm{Cgc}, \mathrm{Gc})$, located in the midline connecting the adhesion line (CA-CP), which are calculate according to the formulas:

$$
F_{c}=\frac{F_{C A}+F_{C P}}{2} ; C g_{c}=\frac{C g_{C A}+C g_{C P}}{2} ; G_{c}=\frac{G_{C A}+G_{C P}}{2}
$$

Zero frontal stereotactic planes pass through the central point which perpendicular to the line (CA-CP); zero sagittal stereotactic plane passes through a line (CA-CP), perpendicular to the frontal and horizontal planes. Central point of stereotactic coordinates of surgical intervention zone is $M(F, C g, G)$ which is determined according to point $(C)$ which is determined according to anatomical slices data from the brain atlases. CT-slices in zero horizontal plane (Fig. 4a) calculated by frontal (F) and sagittal (Cg) stereotactic coordinates, and the horizontal coordinate $(\mathrm{G})$ is determined by a general slice in sagittal projection (Fig. 4b). The visualization of a sagittal plane $(\mathrm{Cg})$ displaced from point $(\mathrm{M})$ is carried out by a general slice scan on the frontal projection (Fig. 4c). Next, the affine transformation of an intra-cerebral coordinate is performed, target points in CT-images system is carried out by using a parallel displacement and rotation of coordinate axes. The parameters $(1 \mathrm{~F}, 1 \mathrm{Cg}, 1 \mathrm{G})$ determine the distance from point $(\mathrm{M})$ to the reference bone landmarks (at the external bone walls, the contours which are visualized in most clearly) which facilitates linking of coordinates.

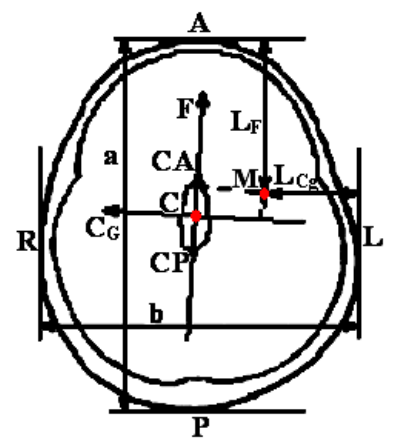

Fig. 4a.

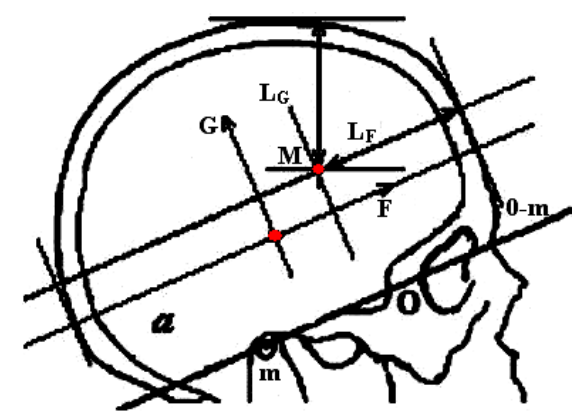

Fig. 4b.

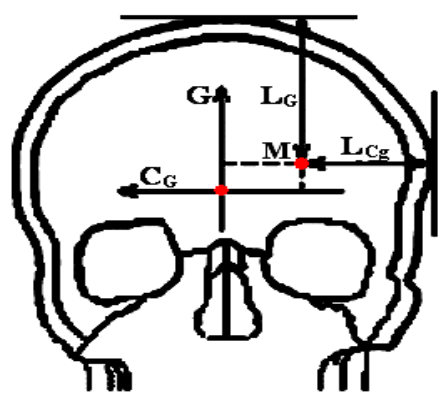

Fig. 4c.

Fig. 4a- Schematic diagram of CT-slice on zero level stereotactic horizontal planes.

Fig. 4b- Schematic diagram of general image in stereotactic sagittal plane.

Fig. 4c- Schematic diagram of general image in stereotactic frontal plane. 
Tareq Alhadidi; Computed Tomography Image-guided Interaoperative and Stereotactic Calculation Method. Journal of Biomedical Engineering and Medical Imaging, Volume 5, No 1, February (2018), pp 1-10

The calculation of surgical instrument orientation based on Kandel stereotactic apparatus. The coordinates of surgical intervention zone is verified by functional control equipment.

\subsubsection{Stereotactic to target movement}

Since the orbitomeatal line parallel to the line (CA-CP) the deviation angle is not more than $\left(5^{\circ}\right)$ [11], horizontal CT-slice which contains the minimum length of local zone (V3) is located in a zero horizontal stereotactic plane (Figure 5c). Zero frontal stereotactic planes passes through the center point perpendicular to a line (CA-CP). Zero sagittal stereotactic planes passes through the line (CA-CP) perpendicular to the frontal and horizontal planes. Stereotactic coordinates for a central point of surgical zone $\mathrm{M}\left(\mathrm{F}^{\prime}, \mathrm{Cg}^{\prime}, \mathrm{G}^{\prime}\right)$ are determined according to point $(\mathrm{C})$, according to anatomical data-slices from brain atlases, and the most perfect of them is the atlas [18]. The determination of individual variability of brain structures can be done by volumetric coefficients. The final stage of surgical intervention zone visualization is the reconstruction of intra-cerebral coordinate zone for target-point into a tomography image coordinates, and that can be achieved by transferring and rotating coordinate's axes, considering the opposite direction of the sagittal axis (see Fig. 5).

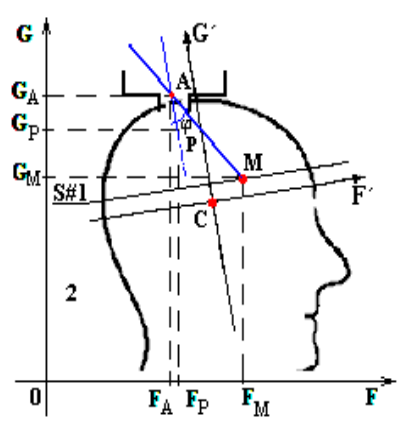

Fig. 5a.

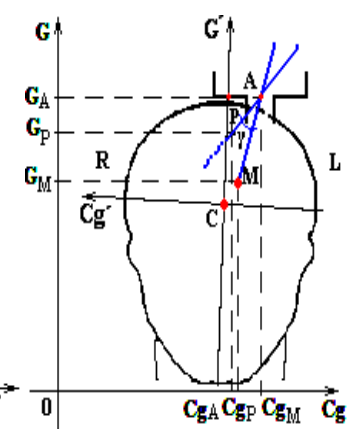

Fig. 5b.

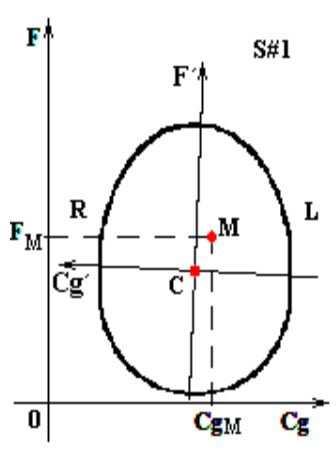

Fig. 5c.

Figure. 5, Surgical-zone visualization (intra-cerebral coordinates zone), and the position of target's point determination.

The calculation of orientation parameters for a surgical instrument movement inside the brain is carried out by a pre-movement with a value of ( $r P \approx 15 \div 20 \mathrm{~mm}$ ) toward the target, and this allows visualization of a CT-plane of the surgical instrument projection in sagittal and frontal planes. Taking into account the known coordinates of the rotation center of the stereotactic apparatus A (FA, CgA,GA) for the target zone $\mathrm{M}(\mathrm{FM}, \mathrm{CgM}, \mathrm{GM})$ and the current position of the distal tip of surgical instrument $\mathrm{P}$ (FP, $\mathrm{CgP}, \mathrm{GP})$ and the rotation angles $(\phi)$ and $(\gamma)$ in the sagittal plane (Fig. 5a) and the frontal plane (Fig. 5b), as well as the surgical instrument movement toward the depth $(r M)$ relative to the point $(A)$, we can use the following formulas:

$$
\begin{gathered}
\operatorname{tg} \varphi=\left|\frac{k_{2}-k_{1}}{1+k_{1} k_{2}}\right|, \operatorname{tg} \gamma=\left|\frac{k_{4}-k_{3}}{1+k_{4} k_{4}}\right| \\
r_{M}=\sqrt{\left(F_{A}-F_{M}\right)^{2}+\left(C g_{A}-C g_{M}\right)^{2}+\left(G_{A}-G_{M}\right)^{2}}
\end{gathered}
$$

Where: 
$\mathrm{k} 1, \mathrm{k} 2, \mathrm{k} 3, \mathrm{k} 4$ - the angular coefficients of straight lines connecting between the current position of surgical instrument distal tip and target in relation to the rotation center of the stereotactic apparatus:

$$
\begin{array}{cc}
k_{1}=\frac{G_{A}-G_{M}}{C g_{A}-C g_{M}}, & k_{2}=\frac{G_{A}-G_{P}}{C g_{A}-C g_{P}} \\
k_{3}=\frac{G_{A}-G_{M}}{F_{A}-F_{M}}, & k_{4}=\frac{G_{A}-G_{P}}{F_{A}-F_{P}}
\end{array}
$$

Thus the additional depth movement of the surgical instrument $(\mathrm{rX})$ taking into account the preliminary depth inside the brain, is:

$$
r_{X}=r_{M}-r_{P}
$$

\section{Results and discussion}

The ability of calculation of additional depth movement for the surgical instrument has extreme importance especially when dealing with a multiple surgical guidance. Thus to apply the method, a software was designed to allow visualization of surgical-intervention zone and for formation of the control parameters of stereotactic apparatus in a surgical instruments guidance.

While using the surgical planning method which applied on 27 patients, the total error of a surgical instrument targeting was less than $3 \mathrm{~mm}$ (in average $2.6 \mathrm{~mm}$ ) compared with others systems. Stereotactic calculations were performed using a developed, calculating graphical software that allows visualization of zone of surgical intervention and forming the control parameters of stereotactic apparatus for guiding the surgical instruments. An intra-operation by using CT-stereotactic calculations applying stimulating electrode into the sub-thalamic zone located $2 \mathrm{~mm}$ laterally, and $10 \mathrm{~mm}$ posterior and $3 \mathrm{~mm}$ ventral from center $(\mathrm{F}=-1.2, \mathrm{Cg}=10.91, \mathrm{G}=-6.23)$ sub-thalamic nucleus: $\mathrm{CT}$-slice at stereotactic zero-plane is shown on (Fig. 6a):

1 - The calculation of coordinates at central surgical zone,

2 - Shot-screen for cannula movement in CT-slice forward surgical intervention zone (Fig. 6b),

3 - Distal point of cannula at the active part.

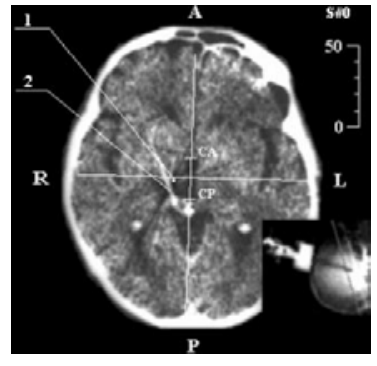

Fig. 6 a.

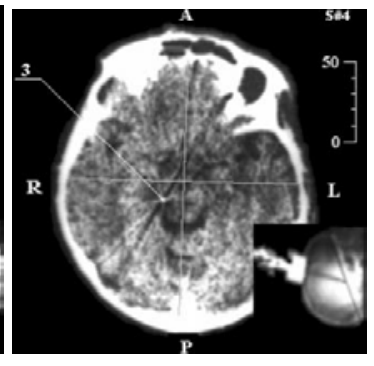

Fig. 6b.

Figure. 6- Demonstration of neurosurgery CT-stereotactic calculation, while targeting the med-nucleus with colorless ball: a). stereotactic CT-slice at zero-plane level, b). CT- stereotactic calculation at the level of surgical zone: 1 . The calculation of central point stereotactic-target zone, 2. Artifact of cannula movement, 3. Distal point of the cannula moving part. 
Tareq Alhadidi; Computed Tomography Image-guided Interaoperative and Stereotactic Calculation Method. Journal of Biomedical Engineering and Medical Imaging, Volume 5, No 1, February (2018), pp 1-10

\section{Conclusions}

The aim of this work is to develop a virtual model of the human brain that could be used in a neurosurgical simulation for preoperative planning purposes. In order to obtain a realistic and useful simulation we focused our study on the physical modeling of the brain targeting (med-nucleus colorless body) and on the maneuvers of the surgical instruments. The study included the following:

1. The study methodology provides high accuracy to identify orbit meatal plane for the skull which is necessary for reference stereotactic landmark determination.

2. The visualization of (V3) contours which is necessary to localize the center of intra-cerebral stereotactic coordinate system.

3. Using the proposed method, it clinically increased the accuracy of stereotactic operations in parkinsonian disease by (30\%).

4. Furthermore, increasing the accuracy of stereotactic-guidance in functional surgery is associated with increasing of the accuracy of ability of the reconstruction techniques of CT-image for obtaining 3D-image and the development of algorithms machine of intro-scope data analysis for full-automatic recognition of the intra-cerebral landmarks taking into account their variation. Also the surgical operations planning system must be improved to allow performing virtual volumetric simulation of the main stages of surgical intervention.

\section{REFERENCES}

[1] Carroll, William M.. International Neurology. John Wiley \& Sons. p. 188. ISBN 9781118777367. Archived from the original on 8 September 2017.

[2] Fingar KR, Stocks C, Weiss AJ, Steiner CA (December 2014). Most Frequent Operating Room Procedures Performed in U.S. Hospitals, 2003-2012"

[3] Sveinbjornsdottir, S . The clinical symptoms of Parkinson's disease. Journal of Neurochemistry. 139:p 318-324. https://www.ncbi.nlm.nih.gov/pubmed/27401947

[4] Fitzgerald, M; Bellgrove M; Gill, M. Handbook of Attention Deficit Hyperactivity Disorder. John Wiley \& Sons. p. 270.

[5] Santosh, Paramala J; Henry, Amy; Varley, Christopher K (24 January 2008). "ADHD and hyperkinetic disorder". In Peter Tyrer; Kenneth R. Silk. Cambridge Textbook of Effective Treatments in Psychiatry. Cambridge University Press. p. 782. ISBN 978-1-139-46757-5.

[6] Zernov D. L'encephalometre. Rev Gen Clin Ther 1890; 19:302.

[7] Carlson, Neil R."Physiology of Behavior".Pearson Education, Inc., 2013. p.134..

[8] Paddick, I. A simple dose gradient measurement tool to complement the conformity index. Journal of Neurosurgery. P 105: 194-201. PMID 18503356. https://www.ncbi.nlm.nih.gov/pubmed/18503356

[9] De Salles, A. Radiosurgery from the brain to the spine: 20 years' experience. Acta Neurochirurgica Supplements. 101: p 163-168. https://www.ncbi.nlm.nih.gov/pubmed/18642653 
Journal of Biomedical Engineering and Medical Imaging, Volume 5, No 1, February 2018

[10] Kavanagh, B. D.. Extracranial radiosurgery (stereotactic body radiation therapy) for oligometastases. Seminars in radiation oncology. $200616(2)$ : p $\quad$ 7784. https://www.ncbi.nlm.nih.gov/pubmed/16564443

[11] Verellen, D. Innovations in image-guided radiotherapy". Nature Reviews. Cancer. 7 (12):p 949-960.

[12] Shershever A. et al. Calculation of target destruction using MRI, during stereotactic-endoscopic microsurgical techniques. Journal of neurosurgery issues. Institute of Neurosurgery named after academician N.N.Burdenko - 2001. - №3. - p:24-25.

[13] Grunert P., Darabi K., Espinosa J., Filippi R, Computer-aided navigation in neurosurgery, Neurosurgery. Rev. - 2003. - V.26, N2. - P.73-99. https://www.ncbi.nlm.nih.gov/pubmed/12962294

[14] Han J.K., Hwang P.H., Smith T.L. Contemporary use of image-guided systems, Curr. Opin. Otolaryngol. Head Neck Surg, 2003. - V.11, N1. - P.33-36. https://www.ncbi.nlm.nih.gov/pubmed/14515099

[15] Iacopino D.G, Conti A, Angileri F.F, Tomasello F, Different methods for anatomical targeting, Journal of Neurosurgery Science. 2003..47, P.18-25. https://www.ncbi.nlm.nih.gov/pubmed/12900728

[16] Athos, J. and Storm, DR. High Precision Stereotaxic Surgery in Mice. Current Protocols in Neuroscience. 2001. A.4A.1-A.4A.9. https://www.ncbi.nlm.nih.gov/pubmed/18428449

[17] Nakao N., Nakai K., Itakura T. Updating of Neuro-navigation based on images intra-operatively acquired with a mobile computerized tomographic scanner. Technical note, Minim. Invas. Neurosurg. 2003 V.46, N2 P.117-120. 